\title{
Treatment Adherence and Abandonment in Acute Myeloid Leukemia in Pediatric Patients at a Low-Resource Cancer Center in India
}

\begin{abstract}
Aim: One of the causes for lower cure rates in acute childhood leukemia in low- and middle- income countries (LMIC) compared to high-income countries is abandonment from treatment. The International Society of Pediatric Oncology (SIOP) defines abandonment as failure to begin treatment or an absence of 4 weeks during treatment. The aim of this study was to evaluate the extent of abandonment among patients diagnosed with acute myeloid leukemia (AML) at the pediatric ward at a low-resource cancer center in India. Methods: Medical records of all patients, aged 0-15 years, diagnosed with AML between January 1, 2014, and March 31, 2015, at the hospital were reviewed. Age, sex, date of diagnosis, and survival during the short follow-up time after completed treatment and information regarding abandonment were collected. SIOP definition of abandonment was used. Eight patients were diagnosed with AML at the hospital whereof 65 met the inclusion criteria of this study. Results: Of the included 65 patients, 6 died before treatment could be initiated and 3 were referred to palliative care upfront. Thus, 56 patients were offered curatively intended treatment. Of these patients, six refused treatment at this stage and another five abandoned during therapy. Altogether, 11 children abandoned treatment. Conclusion: In this study, the abandonment rate from treatment of childhood AML was $20 \%$, which is in concordance from other studies conducted in India and other LMIC, stating that abandonment is a problem and hindrance when treating with a curative intent.
\end{abstract}

Keywords: Abandonment to treatment, acute childhood leukemia, Low middle income countries

\section{Introduction}

In India and other low- and middle-income countries (LMIC), the incidence of cancer in children aged 0-15 years is reported to be $38-124 /$ million children, whereas in high-income countries (HIC), the incidence is $140 /$ million. ${ }^{[1-3]}$ The reported low incidence in India is believed to be the result of underreporting and underdiagnosis. ${ }^{[4]}$ As only $8 \%$ of Asia's population are covered by cancer registries, ${ }^{[5,6]}$ the lack of regional and national cancer registries makes comparison between LMIC such as India and HIC practically impossible. An improved health care systems, and thus a rising number of children surviving to an age when cancer is presented as well as higher awareness of cancer will increase the amount of reported cases of pediatric cancer. ${ }^{[2,7]}$

The survival rates in pediatric cancers in HIC have risen during the past decades to approximately $80 \%$. In LMIC, the cure rate is approximately $10 \%-30 \% \cdot{ }^{[8]}$ The

This is an open access journal, and articles are distributed under the terms of the Creative Commons Attribution-NonCommercial-ShareAlike 4.0 License, which allows others to remix, tweak, and build upon the work non-commercially, as long as appropriate credit is given and the new creations are licensed under the identical terms.

For reprints contact: reprints@medknow.com majority (80\%) of all childhood cancer patients reside in LMIC. Thus, 80\%-90\% of all childhood cancer deaths are in LMIC. ${ }^{[3,5,6]}$ The survival rates of childhood cancers in India are consistently reported to be lower than in HIC. Several factors are reported to correlate with survival, and government health expenditure is one of the most important. ${ }^{[9]}$ In 2013, India spent $1.3 \%$ of total gross domestic product on public health care, which is $<10 \%$ of the expenditures in HIC. ${ }^{[10]}$

The Indian Pediatric Hematology-Oncology Group and International Society of Pediatric Oncology (SIOP) reports that the lower survival rates in LMIC and India specifically mainly are caused by underdiagnosis and abandonment from treatment. ${ }^{[7,11]}$

\section{Abandonment}

SIOP defines abandonment as refusal to begin curatively intended treatment after diagnosis or abandonment after

\footnotetext{
How to cite this article: Sinha S, Brattström G, Palat G, Rapelli V, Segerlantz M, Brun E, et al. Treatment adherence and abandonment in acute myeloid leukemia in pediatric patients at a lowresource cancer center in India. Indian J Med Paediatr Oncol 2019;40:501-6.
}

\section{Sudha Sinha1*, Gustav Brattström ${ }^{2 *}$, Gayatri Palat ${ }^{3,4,5}$, Vineela Rapelli ${ }^{6}$, Mikael Segerlantz ${ }^{7,8}$, Eva Brun ${ }^{9,10}$, Thomas Wiebe ${ }^{11}$}

${ }^{1}$ Department of Medical Oncology, ${ }^{3}$ Palliative Access (PAX) Program, ${ }^{5}$ Department of Pain and Palliative Medicine, ${ }^{6}$ Palliative Care Program, MNJ Institute of Oncology and Regional Cancer Centre, Hyderabad, Telangana, India, ${ }^{2}$ Faculty of Medicine, University of Lund, Lund, Sweden, ${ }^{4}$ Two Worlds Cancer CollaborationINCTR, Vancouver, British Columbia, Canada, ${ }^{7}$ Department of Clinical Sciences, Oncology and Pathology, Institute for Palliative Care, Faculty of Medicine, Lund University, ${ }^{8}$ Department of Palliative Care and Advanced Home Health Care, Primary Health Care Skane, Region Skane, ${ }^{9}$ Department of Clinical Sciences, Oncology and Pathology, Faculty of Medicine, Lund University, ${ }^{10}$ Department of Radiotherapy and Radiophysics, Skane University Hospital, " Department of Clinical Sciences, Paediatrics, Faculty of Medicine, Lund University, Lund, Sweden

*The first two authors have contributed equally to this work

Submitted: 13-Apr-2018

Revised: 16-Aug-2018

Accepted: 18-Oct-2018 Published: 17-Feb-2020

Access this article online Website: www.ijmpo.org DOI: 10.4103/ijmpo.ijmpo_84_18 Quick Response Code:

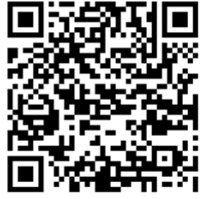


Address for correspondence:

Dr. Mikael Segerlantz,

Palliative Care and Advanced Home Health Care, Sankt Lars Väg 90, 22185 Lund, Sweden. E-mail: mikael.segerlantz@skane.se

initiated treatment or absence of 4 weeks or more during treatment. ${ }^{[12]}$ The latter because such a gap will make cure less probable. Abandonment mainly occurs during the $1^{\text {st }}$ month of treatment. ${ }^{[1,13,14]}$ The reasons for abandonment and treatment refusal seem to be similar and are therefore relevant to address as a single phenomenon, ${ }^{[12]}$ hereafter referred to as abandonment.

According to SIOP and studies made in LMIC, abandonment is one of the major contributors to low survival rates in the cohort of children that actually are offered curatively intended treatment. ${ }^{[2,12,15-18]}$ A review article reports that the abandonment rate (AR) in pediatric acute myeloid leukemia (AML) in India is $19 \%-50 \%,{ }^{[19]}$ figures that must be handled with caution due to the reasons stated above.

Apart from causing dismal survival rates, abandonment also increases unnecessary suffering by disrupted and thus futile cytotoxic treatments. ${ }^{[18]}$

In HIC, abandonment is a nearly nonexisting phenomenon as authorities will intervene in these cases to secure treatment adherence in order to protect the child, ensuring the possibility for cure.

\section{Acute myeloid leukemia}

Childhood leukemia comprises $30 \%$ of all pediatric cancers. The most common form is acute lymphatic leukemia (ALL) and the second most common form (10\% to $20 \%$ of childhood leukemia) is AML. ${ }^{[20]}$

The survival rates of AML in HIC have improved since the 1980 s, from $0 \%$ to $60 \%$ today, ${ }^{[21]}$ when treatment protocol using a combination of anthracyclines and cytarabine was introduced. Intensification of the protocol and improvement of the supportive care have contributed to improved survival. Indian studies report a 5-year survival of 33\% in leukemia in total and $23 \%-58 \%$ for AML, ${ }^{[2,19]}$ for those who are offered curative treatment. Due to the lack of cancer registries in India, these figures only represent selected populations and are not representative for the entire population.

AML is commonly presented with symptoms such as fever, fatigue, bleeding, and bone and joint pain. Leukopenia, thrombocytopenia, and anemia are common laboratory findings. Patients diagnosed with AML are stratified into risk groups according to the French-American-British classification and certain cytogenetic abnormalities. ${ }^{[20]}$

The standard treatment protocol for AML in India consists of two phases. The first phase is the induction phase which lasts for 1 month. This is followed by the consolidation phase which is divided into three parts, each lasting for 1 month, resulting in a total treatment time of 4 months. ${ }^{[19]}$
Aim

The purpose of this study was to evaluate the extent of abandonment in AML treatment, at a low-resource hospital in India, among patients offered curative treatment.

\section{Methods}

In this retrospective study, medical records at the Mehdi Nawaj Jung Institute of Oncology and Regional Cancer Centre (MNJ) were reviewed. Children and adolescents at the age of 15 or less, diagnosed with AML between January 1, 2014, and March 31, 2015, were included in the study. This period ensured at least a 4-month follow-up period after completed treatment. Cases were collected from the medical archives as well as from the bone marrow aspiration (BMA) registry to find as many patients as possible. The patient data were collected concerning age, parental income, sex, date of registration and diagnosis, start of induction treatment and consolidation phase, remission status postinduction, date of completed treatment, survival status and date of death as well as information on abandonment and also referral to the palliative care unit.

The medical records were collected by social workers at the pediatric department at the hospital. A few records were never found. The reason for this could be that medical records are kept by the patients which may result in losses when changing departments or shifting hospitals. Approval was granted for this study by the Institutional Ethics Committee at MNJ.

\section{Abandonment}

Information on treatment and abandonment was retrieved by comparing the recorded events in the patient's medical record to the AML treatment protocol used at the hospital. Date of abandonment was recorded.

\section{Exclusion}

Reasons for exclusion from the study were incomplete medical files, wrong diagnosis, or no diagnosis at all. Patients treated in adult wards and patients that did not finish their treatment according to the protocol during the study period were also excluded from the study.

\section{Statistical analysis}

Survival of the patients was analyzed using Kaplan-Meier analysis in MedCalc Statistical Software version 15.11.4 (MedCalc Software bvba, Ostend, Belgium). Date of diagnosis and death were used as events in the analysis. The last date of follow-up represents last date of visit, abandonment, refusal, or referral to the palliative care department for end-of-life care, and in the 
latter cases considered as date of death, as only a short period of survival is expected.

\section{Results}

\section{Study sample}

From the BMA registry and the files collected from the pediatric oncology ward, 80 children under the age of 15 , diagnosed with AML, admitted to the pediatric ward at the hospital between January 1, 2014, and March 31, 2015, were identified. Eight children were excluded due to missing or incomplete medical records and five were excluded because treatment was not finished within the study timeframe. One patient was excluded as this was a case of relapsed AML. At last, one patient was also excluded as he had not completed treatment within the expected time frame, during the sampling period. The study sample thus included 65 children, 24 females and 41 males. Age distribution and subtypes of AML are shown in Figures 1 and 2. All of the patients that abandoned and all of the patients who were referred to the palliative care department were considered dead as of the last date of visit to the pediatric department or date of referral, respectively. This was done, to be able to estimate the survival, as follow-up of these patients was not available.

Out of the 65 patients, 6 died before treatment could be started and 3 were referred to palliative care upfront. Thus, 56 of the patients were finally offered curative treatment. Of these 56 patients, 6 refused treatment and additionally 5 abandoned during treatment. Thus, in total, $11(20 \%)$ of the patients, where curatively intended treatment was finally offered, abandoned treatment. During the induction phase of the treatment, 19 patients died and additionally two patients were referred to palliative care during the induction phase. Thus, 24 of the initial 65 patients completed the 4-month treatment period and were alive 5 months after date of diagnosis [Figure 3].

The mean follow-up time was 4 months (0-19) from date of diagnosis. None of the children adhering to treatment were lost to follow-up.

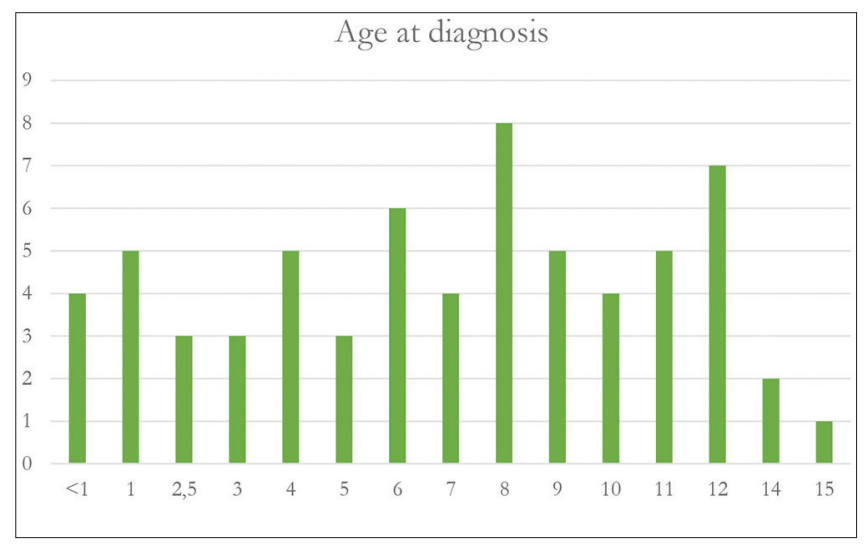

Figure 1: Age at diagnosis in the study population

\section{Abandonment}

Abandonment occurred in a total of 11 of the 56 patients $(20 \%), 6$ girls and 5 boys. None of the patients who abandoned returned to the hospital for further treatment. Out of 11 abandoned patients, 6 were refusals. Four of the abandoning patients did so during the induction phase of the treatment and the remaining one during the consolidation phase.

\section{Survival}

Out of 65 patients, $24(36.9 \%)$ lived 5 months after the date of diagnosis. Of the 56 children, where curative treatment was intended, $43 \%$ lived after 5 months, and for the 50 patients actually starting treatment, the survival rate at 5 months postdiagnosis was $48 \%$. This is displayed in Kaplan-Meier curves [Figures 4-6].

\section{Discussion}

\section{Abandonment rate}

The results in this study are in accordance with other studies from LMIC when treating children with cancer. The AR in this study, $20 \%$, is in the lower range of earlier reported for pediatric AML in India being 19\%-50\%. ${ }^{[19]}$ The AR in our study is also lower compared to AR, for all types of childhood cancer, in other LMIC such as in Kenya (54\%), Zambia (45\%), and Indonesia (48\%). ${ }^{[13,16,22]}$ All studies were using the SIOP definition of abandonment. The underlying causes for abandoning treatment are multifactorial. In an Indonesian study, the most reliable prognostic factor of the outcome of childhood cancer was the parent's socio-economic status. ${ }^{[16]}$ A Zambian study stated that long travel distance to the hospital and low educational level of the mother significantly correlated with a higher risk of abandonment. ${ }^{[22]}$ In families with limited economical resources, loss of income is a major obstacle for staying with a sick child in the hospital. The unawareness that cancer is treatable and curable, the lack of understanding of the underlying reasons for the unavoidable side-effects of the cancer treatment are all factors that will contribute to the families decision

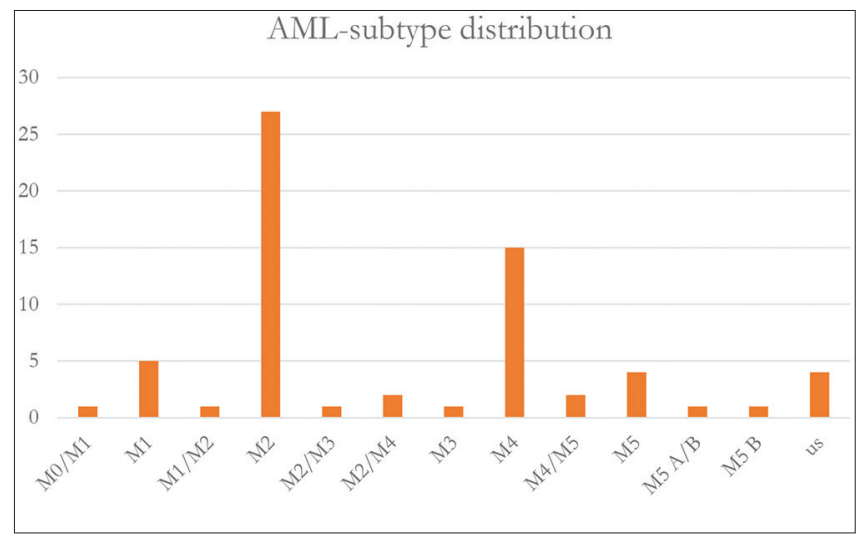

Figure 2: Acute myeloid leukemia subtype in the study population 


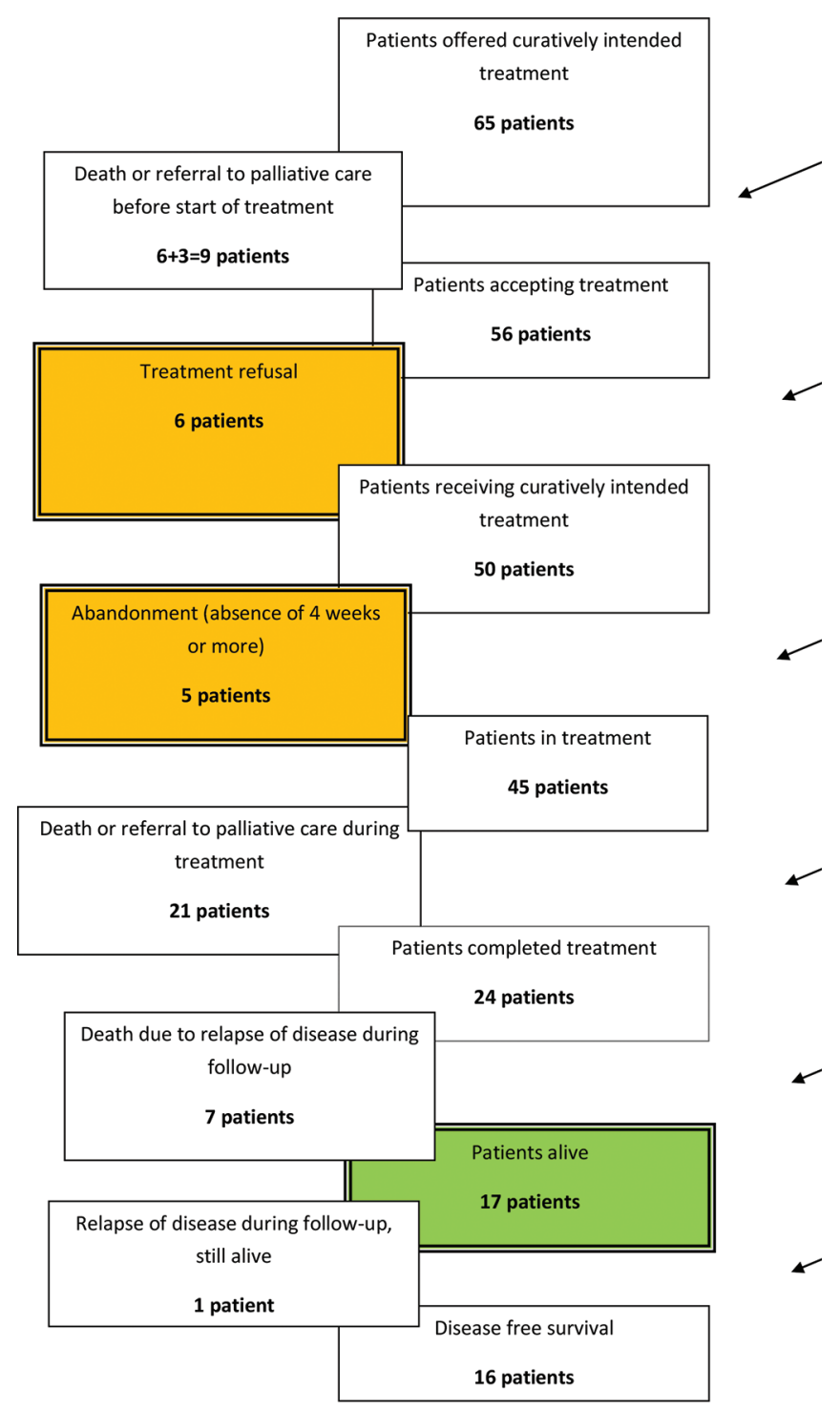

Figure 3: Flow of events in the study population

of abandonment. ${ }^{[1,2,23,24]}$ In the present study, three of the five families abandoning treatment stated a wish to seek treatment elsewhere. Given the poor financial status, this was considered improbable.

In two earlier master theses from Lund University studying abandonment at the studied hospital, the AR for AML in children was 24\% and for ALL and it was 37.5\% (2012 and 2013, unpublished data). The results of these studies have initiated interventions against abandonment, partly evaluated with the present study. During the last years, four additional pediatric oncologists have been recruited to the present hospital. Additional counselors, six in total, each focusing on specific diagnosis, have been employed to support and aid the families. Counselors, among other things, inform the parents about the planned curatively intended treatment and emphasize the importance of treatment adherence. The number of nurses on duty

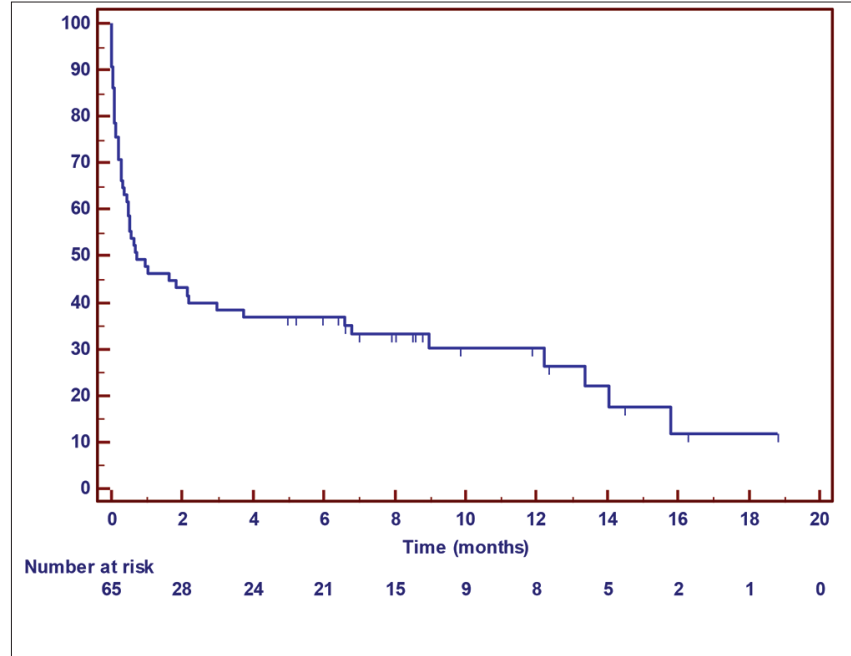

Figure 4: Kaplan-Meier analysis of overall survival of the 65 patients. Note that reason for censoring is either death, abandonment, or referral to the palliative care unit

has increased. Blood tests and treatment with blood products are now possible all hours, all days of the week. Furthermore, a social worker has been hired to keep track of all medical files. The pediatric oncology ward at the present hospital has also developed cooperation with a non-governmental organization which provides financial support for salaries for counselors, transportation support for the families with sick children, and also the costs for blood products.

\section{Survival}

In our study, 24 out of 65 children (37\%) were alive 5 months after diagnosis. In a review article by Kulkarni and Marwaha, ${ }^{[19]}$ overall survival in AML in India ranges between $25 \%$ and $53 \%$. In this study, we found that 21 children out of 50 who actually started curatively intended treatment died at the oncology department or were referred to the palliative care department for end-of-life care. Most of these deaths or referrals happened during the induction phase. This high number of deaths during induction suggests that this treatment phase might be too toxic taken into account the limited resources for supportive care of treatment of side effects. As children that abandoned treatment were lost for follow-up, the last date of visit was counted for as date of death, to enable statistical analysis of survival. We considered it unlikely that the families could finance a complete AML treatment elsewhere. Likewise, the referral of children to palliative care was counted for as date of death in the statistical analysis.

\section{Underdiagnosis and gender}

With $29.1 \%$ of India's population between 0 and $15,{ }^{[25]}$ there are a total of 25.5 million children aged $0-15$ years in the catchment area of this hospital, MNJ. The worldwide incidence of pediatric AML is 6/million children which would result in 153 pediatric AML cases annually in 


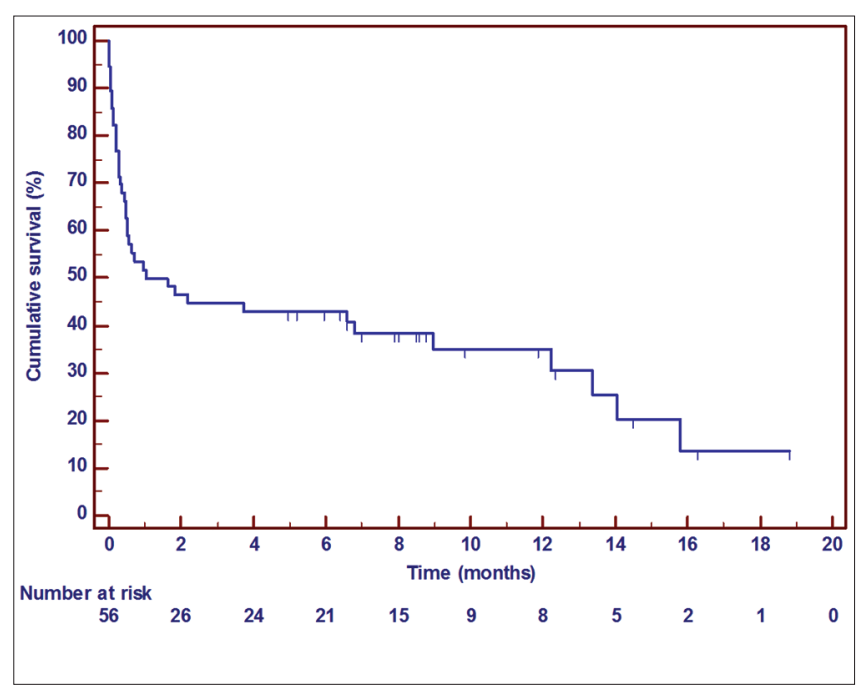

Figure 5: Kaplan-Meier analysis of overall survival of the 56 patients intended for curative treatment. Note that reason for censoring is either death, abandonment, or referral to the palliative care unit

the catchment area of this governmental tertiary cancer center in India. The present hospital treats approximately $70 \%$ of the total number of pediatric cancer patients in the region. ${ }^{[25,26]}$ Thus, we can calculate that the pediatric oncology department at the hospital should see 107 new children with AML each year. During the study period, January 2014 to March 2015, 65 new cases of pediatric AML were registered at the hospital, equaling 52 AML patients in 1 year. This is about half of the estimated number; thus, a situation of underdiagnosis is evident. ${ }^{[26]}$ In the study group, there is an overweight of male patients $(63 \%)$ compared to the female patients. Childhood cancer incidence in India is repeatedly reported to be higher for boys than for girls. During the last decades, the incidence of girls with cancer has increased. Still, there is a great difference in reported incidence not motivated by biological differences between the sexes.

\section{Conclusion}

Abandonment and treatment refusal is a problem at the pediatric ward at the present hospital contributing to unnecessarily high mortality and morbidity. Interventions such as digital medical records, more extensive counseling, and better follow-up have already been implemented to decrease the AR. Nevertheless, continuous efforts are required, as well as actions against the high number of deaths during induction phase of treatment found in this study.

The reasons for refusal and treatment abandonment were only known in a few of the cases in this study. By further investigating the reasons for abandonment, the hospital will be able to further implement required and targeted interventions to minimize the number of patients abandoning or refusing treatment. Underlining the impact of abandonment on treatment outcome in pediatric cancer

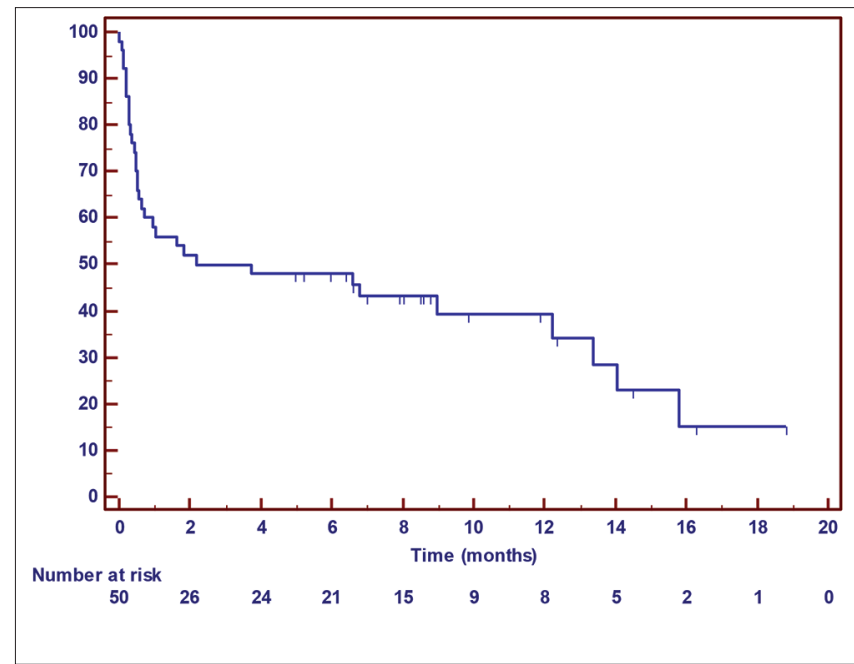

Figure 6: Kaplan-Meier analysis of overall survival of the 50 patients starting curative treatment. Note that reason for censoring is either death, abandonment, or referral to the palliative care unit

may result in higher number of patients completing treatment, thereby increasing survival rates.

Aside abandonment and early treatment deaths, one must conclude that the major cause of mortality in childhood AML in LMIC is that only half of the estimated cases are diagnosed and thus can be offered treatment.

\section{Acknowledgment}

We wish to thank MNJ, Hyderabad, for providing resources and the patients included in the study and their families, in the interest of future patients and families.

\section{Financial support and sponsorship}

Nil.

\section{Conflicts of interest}

There are no conflicts of interest.

\section{References}

1. Arora RS, Eden T, Pizer B. The problem of treatment abandonment in children from developing countries with cancer. Pediatr Blood Cancer 2007;49:941-6.

2. Arora RS, Eden TO, Kapoor G. Epidemiology of childhood cancer in India. Indian J Cancer 2009;46:264-73.

3. Rodriguez-Galindo C, Friedrich P, Alcasabas P, Antillon F, Banavali S, Castillo L, et al. Toward the cure of all children with cancer through collaborative efforts: Pediatric oncology as a global challenge. J Clin Oncol 2015;33:3065-73.

4. Swaminathan R, Sankaranarayanan R. Under-diagnosis and under-ascertainment of cases may be the reasons for low childhood cancer incidence in rural India. Cancer Epidemiol 2010;34:107-8.

5. Ferlay J, Shin HR, Bray F, Forman D, Mathers C, Parkin DM, et al. Estimates of worldwide burden of cancer in 2008: GLOBOCAN 2008. Int J Cancer 2010;127:2893-917.

6. Rodriguez-Galindo C, Friedrich P, Morrissey L, Frazier L. Global challenges in pediatric oncology. Curr Opin Pediatr 2013;25:3-15. 
7. Demanelis K, Sriplung H, Meza R, Wiangnon S, Rozek LS, Scheurer ME, et al. Differences in childhood leukemia incidence and survival between Southern Thailand and the United States: A population-based analysis. Pediatr Blood Cancer 2015;62:1790-8

8. Magrath I, Steliarova-Foucher E, Epelman S, Ribeiro RC, Harif M, Li CK, et al. Paediatric cancer in low-income and middle-income countries. Lancet Oncol 2013;14:e104-16.

9. Ribeiro RC, Steliarova-Foucher E, Magrath I, Lemerle J, Eden T, Forget $\mathrm{C}$, et al. Baseline status of paediatric oncology care in ten low-income or mid-income countries receiving my child matters support: A descriptive study. Lancet Oncol 2008;9:721-9.

10. The World Bank Group - Health Expenditure, Public (\% of GDP). Available from: http://www.data.worldbank.org/indicator/ SH.XPD.PUBL.ZS. [Last accessed on 2017 Mar 30].

11. Yadav SP, Rastogi N, Kharya G, Misra R, Ramzan M, Katewa $\mathrm{S}$, et al. Barriers to cure for children with cancer in India and strategies to improve outcomes: A report by the Indian pediatric hematology oncology group. Pediatr Hematol Oncol 2014;31:217-24.

12. Mostert S, Arora RS, Arreola M, Bagai P, Friedrich P, Gupta S, et al. Abandonment of treatment for childhood cancer: Position statement of a SIOP PODC working group. Lancet Oncol 2011;12:719-20.

13. Njuguna F, Mostert S, Slot A, Langat S, Skiles J, Sitaresmi MN, et al. Abandonment of childhood cancer treatment in Western Kenya. Arch Dis Child 2014;99:609-14.

14. Ramzan M, Yadav SP, Sachdeva A. Treatment abandonment is a major hurdle to improving survival in childhood cancer in the developing world. Pediatr Blood Cancer 2013;60:159-60.

15. Gupta S, Yeh S, Martiniuk A, Lam CG, Chen HY, Liu YL, et al. The magnitude and predictors of abandonment of therapy in paediatric acute leukaemia in middle-income countries: A systematic review and meta-analysis. Eur J Cancer 2013;49:2555-64.

16. Mostert S, Gunawan S, Wolters E, van de Ven P, Sitaresmi M, Dongen JV, et al. Socio-economic status plays important roles in childhood cancer treatment outcome in Indonesia. Asian Pac J
Cancer Prev 2012;13:6491-6.

17. Spinetta JJ, Masera G, Eden T, Oppenheim D, Martins AG, van Dongen-Melman J, et al. Refusal, non-compliance, and abandonment of treatment in children and adolescents with cancer: A report of the SIOP working committee on phychosocial issues in pediatric oncology. Med Pediatr Oncol 2002;38:114-7.

18. Weaver MS, Arora RS, Howard SC, Salaverria CE, Liu YL, Ribeiro RC, et al. A practical approach to reporting treatment abandonment in pediatric chronic conditions. Pediatr Blood Cancer 2015;62:565-70.

19. Kulkarni KP, Marwaha RK. Childhood acute myeloid leukemia: An Indian perspective. Pediatr Hematol Oncol 2011;28:257-68.

20. de Rooij JD, Zwaan CM, van den Heuvel-Eibrink M. Pediatric AML: From biology to clinical management. J Clin Med 2015;4:127-49.

21. Pui CH, Carroll WL, Meshinchi S, Arceci RJ. Biology, risk stratification, and therapy of pediatric acute leukemias: An update. J Clin Oncol 2011;29:551-65.

22. Slone JS, Chunda-Liyoka C, Perez M, Mutalima N, Newton R, Chintu $\mathrm{C}$, et al. Pediatric malignancies, treatment outcomes and abandonment of pediatric cancer treatment in Zambia. PLoS One 2014;9:e89102.

23. Mostert S, Njuguna F, Langat SC, Slot AJ, Skiles J, Sitaresmi MN, et al. Two overlooked contributors to abandonment of childhood cancer treatment in Kenya: Parents' social network and experiences with hospital retention policies. Psychooncology 2014;23:700-7.

24. Rossell N, Gigengack R, Blume S. Childhood cancer in El Salvador: A preliminary exploration of parental concerns in the abandonment of treatment. Eur J Oncol Nurs 2015;19:370-5.

25. Un data - UN Country Profile: India. Available from: http://www. data.un.org/CountryProfile.aspx?crName=INDIA. [Last accessed on 2017 Mar 30].

26. Sudha Sinha MD, Medical Oncology, MNJ Institute of Oncology and Regional Cancer Centre, Hyderbad, Telungana, India. In: Brattström GM, editor, Medical Student, Hyderabad, India: Lund University; 2015. 